\title{
Marchiafava-Bignami Disease: A Less Known Neurological Complication of Alcoholism
}

\author{
Samuel Fonseca ${ }^{\mathrm{a}, \mathrm{c}}$, Arlindo Guimas ${ }^{\mathrm{b}}$, Diana Valadares ${ }^{\mathrm{b}}$, Graziela Carvalheiras ${ }^{\mathrm{b}}$, \\ Pedro Vita ${ }^{\mathrm{b}}$
}

\begin{abstract}
Marchiafava-Bignami disease (MBD) is a disorder associated with chronic alcohol consumption and characterized by demyelination and necrosis of the corpus callosum. Patients may have reduced consciousness, psychotic symptoms, apathy, hemiparesis, ataxia and coma. Although some cases of recovery have been reported, the outcome is poor, with disability or dead. We reported a case of a 56-year-old woman with chronic alcoholism admitted to our hospital with impaired consciousness and a history of gait disturbance. On examination, she was in a coma, with increased muscle tone and left arm paresis. Laboratory studies showed a folic acid deficiency and the CT scan was normal. Magnetic resonance imaging revealed lesions in periventricular white matter and the entire corpus callosum with restriction of water diffusion consistent with the diagnosis of MBD. Despite treatment with B vitamin complex, the patient died. Coma and radiological involvement of the entire corpus callosum are compatible with type A MBD and associated with poor prognosis. Although being a rare alcoholism complication, it should not be forgotten since an early diagnosis and prompt supportive therapy may enable a better outcome.
\end{abstract}

Keywords: Marchiafava-Bignami disease; Alcoholism; Corpus callosum; Magnetic resonance imaging

\section{Introduction}

Marchiafava-Bignami disease (MBD) was first described in 1903 by the Italian pathologists Amico Bignami and Ettore Marchiafava. It is a disorder associated with chronic alcohol

\section{Manuscript accepted for publication March 24, 2016}

aDepartment of Internal Medicine, Hospital de Santo Antonio, Centro Hospitalar do Porto, Porto, Portugal

beparment of Intensive Care, Hospital de Santo Antonio, Centro Hospitalar do Porto, Porto, Portugal

${ }^{\mathrm{c} C o r r e s p o n d i n g ~ A u t h o r: ~ S a m u e l ~ F o n s e c a, ~ H o s p i t a l ~ d e ~ S a n t o ~ A n t o n i o, ~ C e n-~}$ tro Hospitalar do Porto, Largo Prof. Abel Salazar, 4099-001 Porto, Portugal. Email: samuelbarbosafonseca@hotmail.com

doi: http://dx.doi.org/10.14740/jmc2477w consumption and characterized by demyelination and necrosis of the corpus callosum [1]. In many cases, magnetic resonance imaging (MRI) also reveals hemispheric white matter lesions [2]. Clinically, patients may present with reduced consciousness, emotional and psychotic symptoms, depression and apathy, hemiparesis, ataxia and often coma and death [3].

Before brain imaging was available, the disease was characterized as acute, subacute and chronic [1]. In 2004, Heinrich et al described two clinical subtypes of MBD: type A predominantly characterized by stupor and coma, pyramidal symptoms and radiological involvement of the entire corpus callosum; type B, with slighter impairment of consciousness level and partial or focal lesions of the corpus callosum [1].

In 2001, Helenius et al reported that about 250 new cases have been reported since the original description of the disease, over 200 of them had died, 30 severely demented or bedridden, and only 20 favorably recovered [3]. Nevertheless, there have been some reported cases with clinical and imaging improvement [3-6].

We report a case of acute MBD with extensive brain involvement, severe impaired mental status and poor prognosis.

\section{Case Report}

A 56-year-old female patient was admitted to our institution after being found lying at home with impaired consciousness. She had a history of chronic alcohol intake for about 30 years, with several unsuccessful attempts to alcohol detoxification, and had a gait disturbance for the last week. There was no history of fever, vomiting or seizure, and was last seen the day before in her usual state of health.

On examination, the patient was found to be malnourished and was in a coma, unresponsive to painful stimulation, pupils were of normal size and normal reacting, muscle tone was increased (with the exception of the left arm which was flaccid) and bilateral positive plantar reflex. Signs of meningeal irritation were absent. Laboratory results revealed macrocytosis (105.4 fL) without anemia, normal renal function and normal ion and glucose count. She had a folic acid deficiency (3.8 ng/ $\mathrm{mL}$ ). There was no elevation of C-reactive protein and hepatic liver tests were normal with the exception of gamma-glutamyl transpeptidase (99 U/L). pH was normal although she had an increased anion gap with urinary keto acids. Ethanol was be- 


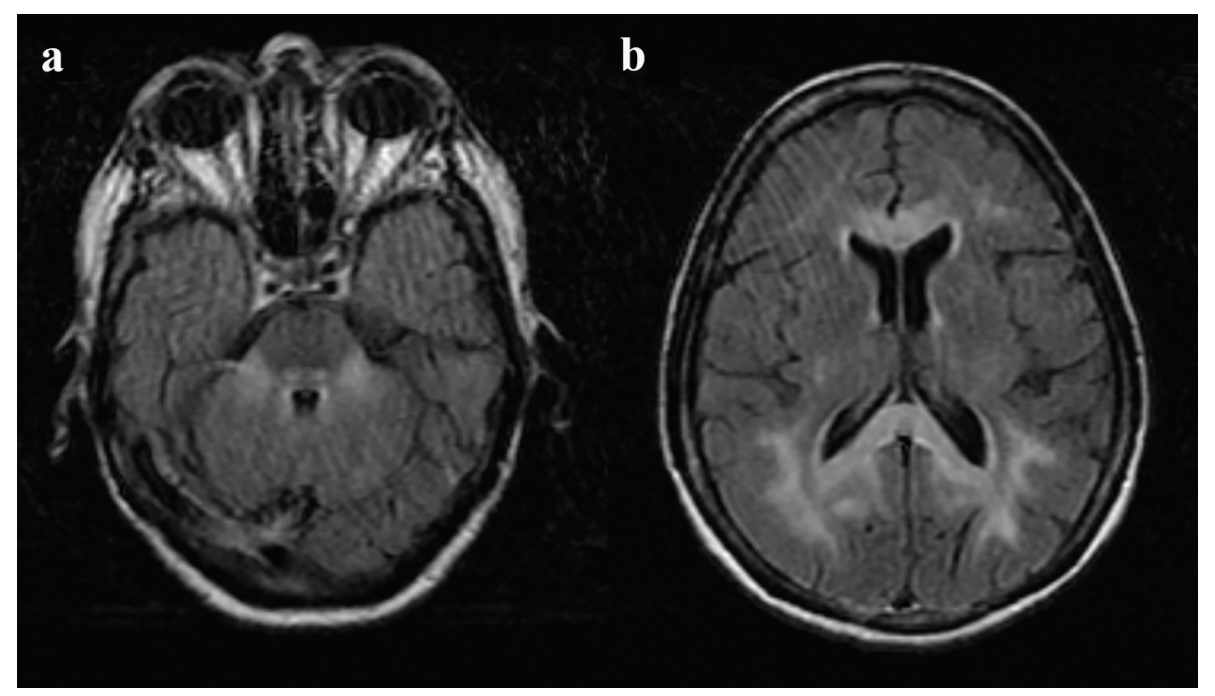

Figure 1. Cerebral MRI, fluid attenuated inversion recovery (FLAIR) image: hyperintense lesion in cerebellar peduncles, pontine tegmentum and central tegmental tracts (a); hyperintense signal in the genu and splenium of the corpus callosum (b).

low $10 \mathrm{mg} / \mathrm{dL}$. Cerebral CT scan showed no lesions and cerebral spinal fluid had normal cell count. She was treated with intravenous fluids, folic acid, thiamine and B complex vitamins.

The cerebral MRI 2 days later showed extensive lesions with hyperintense on long TR sequences involving the cerebellar hemispheres, superior and middle cerebellar peduncles, the pontine tegmentum, the central tegmental tracts and midbrain (Fig. 1). It also revealed the involvement of periventricular subcortical white matter of the cerebral hemispheres and the entire corpus callosum with restriction of water diffusion (Fig. 2 ). Some lesions involved the gray matter, particularly bilaterally precentral cortex and thalamus. On the basis of clinical history and imaging features, the diagnosis of MBD was made.

There was no improvement in neurological status and the patient evolved with hypercapnic respiratory failure. Given the poor prognosis of the disease, the extent of injuries and poor nutritional status of the patient, it was decided not to escalate therapy with ventilatory support. The patient died the fifth day of hospitalization.

\section{Discussion}

MBD is seen most often in individuals with chronic alcoholism; however, some cases have been reported in malnourished patients with no history of alcoholism [7, 8]. Although chronic alcoholism and nutritional deficits are commonly associated with the disease, etiology is still controversial [9].

In our case, the patient had involvement of the entire corpus callosum and presented with coma and pyramidal tract symptoms which are compatible with the A type of MBD, usually associated with poor prognosis $[1,10]$. Treatment with thiamine,

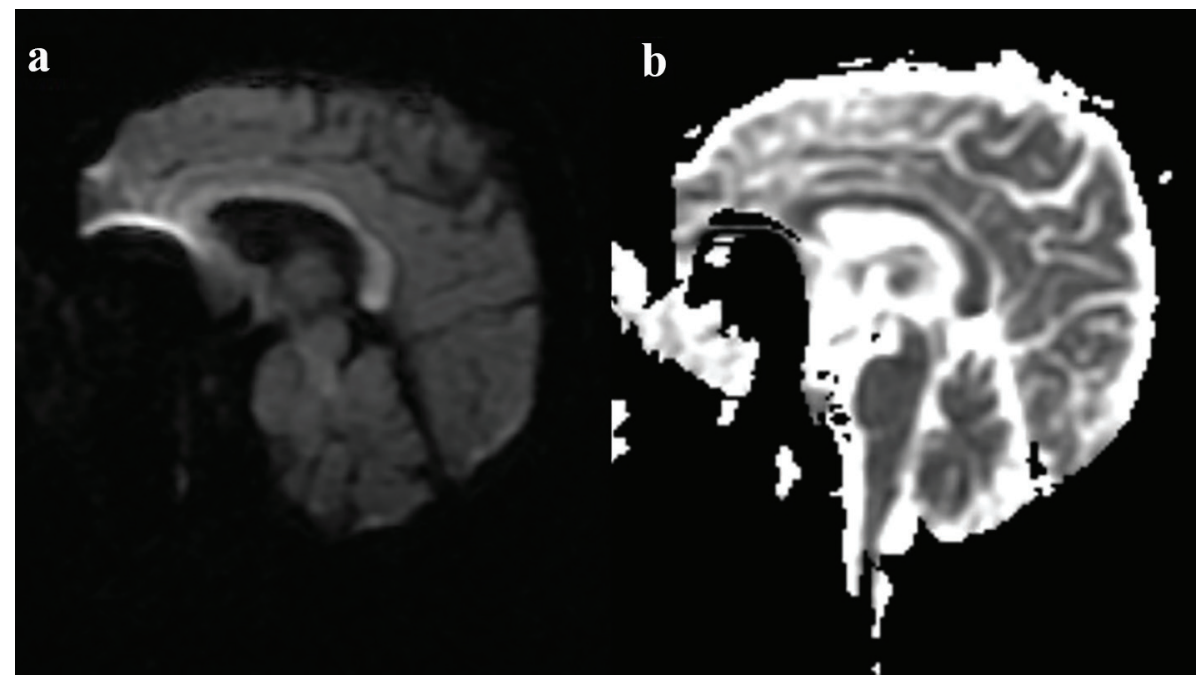

Figure 2. Cerebral MRI, corpus callosum involvement: hyperintense in diffusion-weighted imaging (a) and hypointense on the $\operatorname{ADC}(b)$. 
folic acid and other B complex vitamins has been described in several patients with favorable outcome $[3,4,8]$, and a review of CT/MRI confirmed MBD cases revealed a linear trend for better outcomes among patients treated with thiamine [11]. However, vitamin therapy was also used in cases where there was no clinical recovery $[12,13]$, as in the present case.

MBD is a rare complication associated with alcohol consumption and should not be forgotten. Despite the poor prognosis, an early diagnosis using MRI and prompt supportive therapy may enable a better outcome. Further studies are needed to clarify the etiopathogenesis of the disease and help define an effective treatment.

\section{References}

1. Heinrich A, Runge U, Khaw AV. Clinicoradiologic subtypes of Marchiafava-Bignami disease. J Neurol. 2004;251(9):1050-1059.

2. Ihn YK, Hwang SS, Park YH. Acute Marchiafava-Bignami disease: diffusion-weighted MRI in cortical and callosal involvement. Yonsei Med J. 2007;48(2):321-324.

3. Helenius J, Tatlisumak T, Soinne L, Valanne L, Kaste M. Marchiafava-Bignami disease: two cases with favourable outcome. Eur J Neurol. 2001;8(3):269-272.

4. Diraison P, Rouhart F, Goas P, Auclerc-Guichaoua S, Gomes C, Mocquard Y, Goas JY. [Marchiafava-Bignami disease. 3 cases with favorable prognosis]. Rev Med Interne. 1999;20(1):54-59.

5. Yamashita K, Kobayashi S, Yamaguchi S, Koide H, Nishi K. Reversible corpus callosum lesions in a patient with
Marchiafava-Bignami disease: serial changes on MRI. Eur Neurol. 1997;37(3):192-193.

6. Tung CS, Wu SL, Tsou JC, Hsu SP, Kuo HC, Tsui HW. Marchiafava-Bignami disease with widespread lesions and complete recovery. AJNR Am J Neuroradiol. 2010;31(8):1506-1507.

7. Rickert CH, Karger B, Varchmin-Schultheiss K, Brinkmann B, Paulus W. Neglect-associated fatal MarchiafavaBignami disease in a non-alcoholic woman. Int J Legal Med. 2001;115(2):90-93.

8. Mas G, Gonzalez-Caballero G, Martinez-Ortiz MJ, SaezCastan J. [Marchiafava-Bignami disease in a non-alcoholic patient]. Rev Neurol. 2006;42(10):637-638.

9. Bano S, Mehra S, Yadav SN, Chaudhary V. Marchiafava-Bignami disease: Role of neuroimaging in the diagnosis and management of acute disease. Neurol India. 2009;57(5):649-652.

10. Hlaihel C, Gonnaud PM, Champin S, Rousset H, TranMinh VA, Cotton F. Diffusion-weighted magnetic resonance imaging in Marchiafava-Bignami disease: followup studies. Neuroradiology. 2005;47(7):520-524.

11. Hillbom M, Saloheimo P, Fujioka S, Wszolek ZK, Juvela $\mathrm{S}$, Leone MA. Diagnosis and management of Marchiafava-Bignami disease: a review of CT/MRI confirmed cases. J Neurol Neurosurg Psychiatry. 2014;85(2):168-173.

12. Vazquez C, Salamano R, Legnani C, Cardinal P. [Marchiafava-Bignami disease in Uruguay]. Neurologia. 2008;23(5):322-328.

13. Raina S, Mahesh DM, Mahajan J, Kaushal SS, Gupta D, Dhiman DS. Marchiafava-Bignami disease. J Assoc Physicians India. 2008;56:633-635. 